\title{
ROEDORES FÓSILES DEL PLEISTOCENO SUPERIOR DE LA LOCALIDAD LA PALMERA DE SAN CARLOS, PROVINCIA DE ALAJUELA, COSTA RICA
}

\author{
César Laurito Mora \\ INA, Instituto Nacional de Aprendizaje - Núcleo de Turismo \\ Apdo. Postal 203-2200, Coronado; San José - Costa Rica \\ CLauritoMora@ina.ac.cr
}

(Recibido 30/3/04; aceptado 8/6/04)

\begin{abstract}
A rodent fossil fauna from La Palmera locality at San Carlos area is described. Four new species Tylomys watsoni, Reithrodontomys mexicanus, Sigmodon hispidus and Proechimys semispinosus are reported. A probably Upper Pleistocene age is assigned to this assemblage, because of the paleoflora associated with the rodents, which suggests a more temperate weather, probably a glacial age.
\end{abstract}

Keywords: Fossil mammals, Rodentia, Muridae, biography, paleoecology.

\begin{abstract}
RESUMEN: Se describe una fauna de roedores fósiles de la Localidad de La Palmera de San Carlos. Cuatro nuevas especies de mamíferos: Tylomys watsoni, Reithrodontomys mexicanus, Sigmodon hispidus y Proechimys semispinosus se reportan para Costa Rica; la edad probable de este hallazgo es el Pleistoceno Superior. Esta fauna se encuentra asociada con flora fósil que sugiere al igual que la asociación de roedores un clima mucho más frío, propio de una época glaciar.
\end{abstract}

Plabras clave: Mamíferos fósiles, Rodentia, Muridae, biogeografía, paleoecología.

\section{INTRODUCCIÓN}

Son pocos los registros de roedores fósiles y de micromamíferos registrados para Costa Rica; los únicos hallazgos conocidos fueron reportados por McGroskey (1982) y Grady (1982) ambos citados en Woodman (1988) y los registros presentados por este último autor, todos procedentes de sedimentos del Holoceno medio-tardío (?), extraídos de las cavernas de Barra Honda. El hallazgo más sobresaliente, correspondió al registro de Peromyscus stirtoni, un roedor nunca antes reportado para Costa Rica y que representa una distribución precolombina, en un habitad de bosque seco, el que posteriormente se perdió, debido principalmente al desarrollo de la agricultura (Woodman, 1988).

La localidad de la Palmera de San Carlos, se encuentra en la provincia de Alajuela; coordenadas generales 267-268 N y 495-496 E; hoja topográfica Aguas Zarcas (IGNCR-3347 III) y se ubica a 400 m.s.n.m. (ver mapa de ubicación en Pérez, 2003); de ella se han recuperado una serie de nuevos fósiles, específicamente roedores, 
pero ya había sido previamente estudiada por Pérez, 1998, 2001 y 2003; Pérez \& Laurito, 2003; quienes analizaron su paleobotánica e interpretaron la paleoecología del yacimiento.

El objetivo del presente trabajo es dar a conocer los hallazgos paleovertebradológicos asociados a la flora fósil y a la luz de estos, interpretar la paleoecología, la tafonomía y edad del yacimiento.

\section{MÉTODOS DE RECOLECCIÓN Y LABORATORIO}

La recolección del material se realizó en los depósitos de travertino de La Palmera, depósitos asociados a las fuentes termales de Aguas Zarcas. La cantera que se explota con el fin de producir carbonato de calcio, a menudo es dinamitada, por tal motivo no se tiene un control exacto del nivel estratigráfico del cual procede el bloque que contenía los roedores, pues fueron expelidos luego de una explosión, y se tiene la certeza de que procede de un nivel no superior a los 5 metros sobre el camino que corta la cascada, por lo que correspondería a la zona media del yacimiento.

Los molares y otras piezas dentales y óseas fueron extraídas del bloque mediante el uso de ácido acético al 5\% para remover el carbonato de calcio y la extracción directa utilizando exploradores dentales.

Luego el material fue limpiado, mediante cubetas ultrasónicas y se recubrieron con oro para la obtención de las imágenes de microscopía electrónica, realizadas en la Unidad de Microscopía Electrónica de la Universidad de Costa Rica.

La identificación de las especies, se hizo por comparación anatómica con ejemplares de la colección de roedores del Museo Nacional de Costa Rica y en ella participaron estudiantes del programa de licenciatura de la carrera en Manejo en Recursos Naturales de la Universidad Estatal a Distancia.

El material se encuentra depositado en la Colección de Fósiles de la Sección de Geología del Museo Nacional de Costa Rica.

\section{PALEONTOLOGÍA}

A continuación se describen los aspectos tafonómicos, sistemáticos y paleoecológicos del hallazgo.

\section{Tafonomía}

Los restos proceden de un nido de un ave rapaz y están compuestos por abundantes piezas dentales, principalmente maxilares rotos y mandíbulas parcialmente digeridas, así como restos postcraneános entre los que destacan falanges, húmeros y láminas escapulares, todos de roedores.

Los restos se asocian a bolos fecales y probables bolos de regurgitación y se encuentran asociados a gran cantidad de restos de miriápodos desarticulados. Los miriápodos se han encontrado fosilizados en otros puntos del yacimiento, pero no en la abundancia y concentración de esta nidada en particular. Probablemente tuvieron la función de carroñeros, alimentándose de los restos en descomposición asociados a los bolos fecales o fueron alimento de las aves y sus restos desarticulados estarían asociados a bolos digestivos.

La abundancia de incisivos y molares es común a este tipo de nido, su anormal acumulación obedece a una selección digestiva, en la cual los restos dentales superan el tracto digestivo debido a su mayor dureza (Andrews, 1990).

El nido probablemente se ubicaba en una abertura producto del modelado cársico que afectó el travertino, ello se comprueba fácilmente en el campo, pues son comunes los hallazgos de pequeñas cavernas, algunas de ellas con diámetros de $1,5 \mathrm{~m}$, aunque la mayoría son aberturas decimétricas, con pequeñas estalactitas y velos de calcita que pudieron proveer un abrigo rocoso a aves y otros organismos.

El proceso de fosilización de esta nidada, pudo haber sido muy corto, las aguas termales ricas en carbonato de calcio, rápidamente cementaron los restos, debe tenerse en cuenta que el yacimiento siguió creciendo y posteriores capas de travertino siguieron superponiéndose, proceso que aún hoy continúa, de manera que se preservó el nido y su contenido orgánico. 


\section{Sistemática}

Se describen a continuación cuatro especies de roedores a partir de su patrón dental.

\section{Orden RODENTIA}

Suborden HYSTRICOGNATHI

Infraorden CAVIOMORPHA

Superfamilia OCTODONTOIDEA

Familia ECHIMYIDAE

\section{Proechimys semispinosus}

Material: cuatro molares; figuras 1 a-d.

Descripción: molares de mediano tamaño con longitudes mesiodistales y linguovestibulares cercanas a $1,5 \mathrm{~mm}$, su contorno es ligeramente cuadrangular e inflado, con patrón oclusal sigmoideo característico de los echimyidos.

El CFM-1263 (figura 1a), corresponde al tercer molar inferior derecho $\left(\mathrm{M}_{3}\right)$, con el paracónido y protocónido unidos formando un lófido posterior continuo o metalófido; el hipocónido unido al entocónido por un entolófido; se observa un mesolófido que se esta separado del entolófido por un entofléxido de aspecto periforme.

El CFM-1264 (Fig. 1b), corresponde al segundo molar superior derecho $\left(\mathrm{M}^{2}\right)$ y el CFM1265 (Fig. 1c), corresponde al ${ }^{2} \mathrm{M}$; presentan un contorno cuadrangular, redondeados en la región anteromediana y patrón oclusal sigmoideo, con el paralofo desarrollado; hipocono y metacono unidos por un metalofo y posterolofo bien desarrollado con posterostilos parcialmente desgastados.

El CFM-1266 (Fig. 1d), corresponde a un $\mathrm{M}^{3}$ con similar configuración dental a los anteriores ejemplares, pero presenta menor desgaste, con el paracono intacto y el paralofo parcialmente desarrollado.

Distribución actual: Proechimys semispinosus se distribuye desde el Este de Honduras hasta el Noroeste de Perú y la Amazonia brasileña (Reid, 1997).

En Costa Rica su distribución está confinada, más o menos a las tierras bajas y húmedas del Atlántico y del Pacífico (Llanuras de San

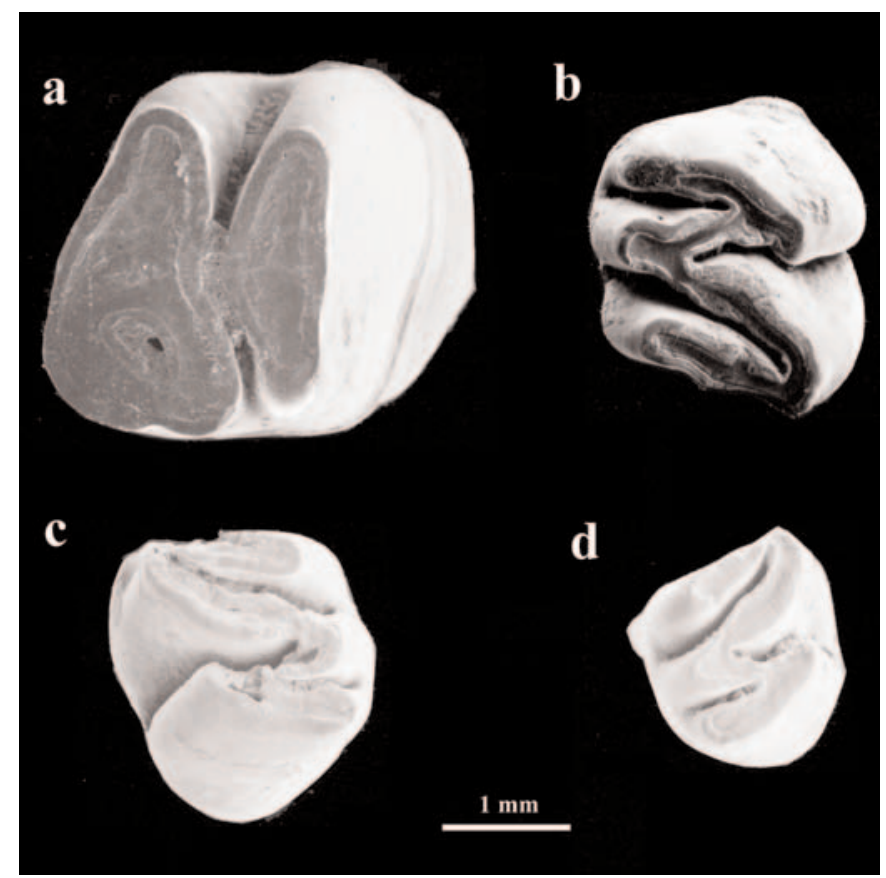

Fig. 1: Molares de Proechimys semispinosus (Tomes, 1860). a: $\mathrm{M}^{3}$ tercer molar inferior derecho; $\mathrm{b}: \mathrm{M}^{2}$ segundo molar superior derecho; c: ${ }^{2} \mathrm{M}$ segundo molar superior izquierdo; $\mathrm{d}: \mathrm{M}^{3}$ tercer molarsuperior derecho. Todos muestran desgaste conspicuo de la superficie oclusal. 
Carlos; Región Caribe y Península de Osa), según Mc Pherson, 1985. En cuanto a zonas de vida, se capturaron ejemplares entre los 0 y 1400 m.s.n.m. en el bosque tropical seco, bosque tropical húmedo, bosque tropical húmedo de transición al premontano hasta el bosque premontano lluvioso, así como bosques basales (Mc Pherson, 1985).

Distribución paleogeográfica: no hay registros de Proechimys semispinosus como especie fósil.

\section{Suborden SCIUROGNATHI Infraorden MYOMORPHA Superfamilia MUROIDEA Familia MURIDAE}

\section{Tylomys watsoni}

Material: dos molares superiores derechos; figuras $2 \mathrm{a}-\mathrm{b}$

Descripción: el CFM-1267 corresponde al M¹ (Fig. 2a), presenta un patrón complejo característico del género Tylomys, donde además de los conos principales se observan los cónulos anterolingual y anterolabial; un anteroflexo y paraflexo separados por el anterolofo largo y delgado; mesoflexo y metaflexo separados por un mesolofo muy desarrollado y delgado; protoflexo e hipoflexo profundos sin protostilo y enterostilo; metalofo y paralofo delgados, posterolofo bien desarrollado, se observan una serie de mesoconos a lo largo del delgado muro mediano.

El CFM-1268 corresponde al $\mathrm{M}^{2}$ (Fig. $2 b)$, con un desgaste incipiente de la superficie oclusal, con mesostilo y parastilo intactos limitados por el mesoflexo y metaflexo, y un hipoflexo respectivamente.

Distribución actual: Tylomys watsoni se distribuye desde México hasta el Oeste de Panamá y desde el Oeste de Colombia hasta el Norte de Ecuador en la región Andina. En Costa Rica habita desde el nivel del mar hasta los 1500 metros de altitud en la vertiente Caribe (incluida la Región Huetar Norte) y el Pacífico Central y Sur, según Mc Pherson, 1985.

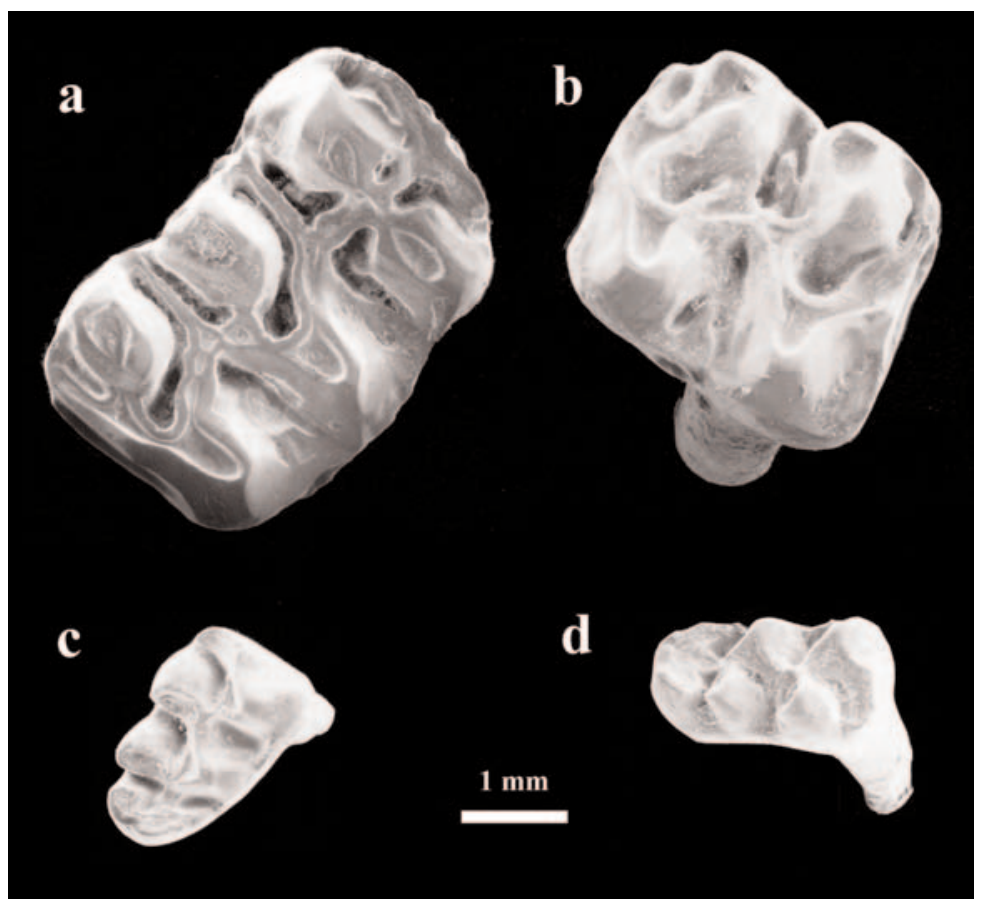

Fig. 2: Molares de Tylomys watsoni y Reithrodontomys mexicanus. a: $\mathrm{M}^{1}$ primer molar superior derecho con desgaste incipiente; b: $\mathrm{M}^{2}$ segundo molar superior derecho sin desgaste de la superficie oclusal; a y b de Tylomys watsoni. c: ${ }^{1} \mathrm{M}$ primer molar superior izquierdo con desgaste incipiente; $\mathrm{d}$ : $\mathrm{M}^{1}$ primer molar superior derecho sin desgaste de la superficie; $\mathrm{c}$ y d de Reithrodontomys mexicanus. 
Distribución paleogeográfica: no hay registros de Tylomys watsoni como especie fósil.

\section{Reithrodontomys mexicanus}

Material: dos molares superiores con la región anteromediana curva, longitud anterioposterior 1,5 $\mathrm{mm}$ y longitud labiolingual $0,6 \mathrm{~mm}$; figuras $2 \mathrm{c}-\mathrm{d}$.

Descripción: el CFM-1269 corresponde al ${ }^{1} \mathrm{M}$ (Fig. 2c) con estadio de desgaste incipiente y el CFM-1270 corresponde al $\mathrm{M}^{1}$ (Fig. 2d) sin desgaste visible.

Ambos ejemplares se distinguen por poseer un prominente hipocono y un posterolofo corto, el paracono, metacono y protocono ligeramente más pequeños que el hipocono; un procíngulo con cónulos anterolingual y labial reducidos y mesocono prominente.

Distribución actual: Reithrodontomys mexicanus se distribuye desde el este y suroeste de México hasta el oeste de Panamá y desde los Andes colombianos hasta Ecuador.

En Costa Rica su rango altitudinal varía desde los 150 m.s.n.m. en Potrero Grande hasta los 1915 m en Tres Colinas, provincia de Puntarenas, pero el rango de mayor ocurrencia está entre 1000 y 1600 m.s.n.m. a lo largo de las cadenas montañosas (Mc Pherson, 1985).

Distribución paleogeográfica: el género Reithrodontomys se ha registrado para el Holoceno Medio-Tardío de la Caverna Ojos Verdes en Barra Honda, Península de Nicoya (Woodman, 1987), no así la especie.

\section{Sigmodon hispidus}

Material: 17 molares con patrón oclusal sigmoideo; figuras 3 a-i.

Descripción: los molares superiores se caracterizan por un ancho posterolofo unido al hipocono,

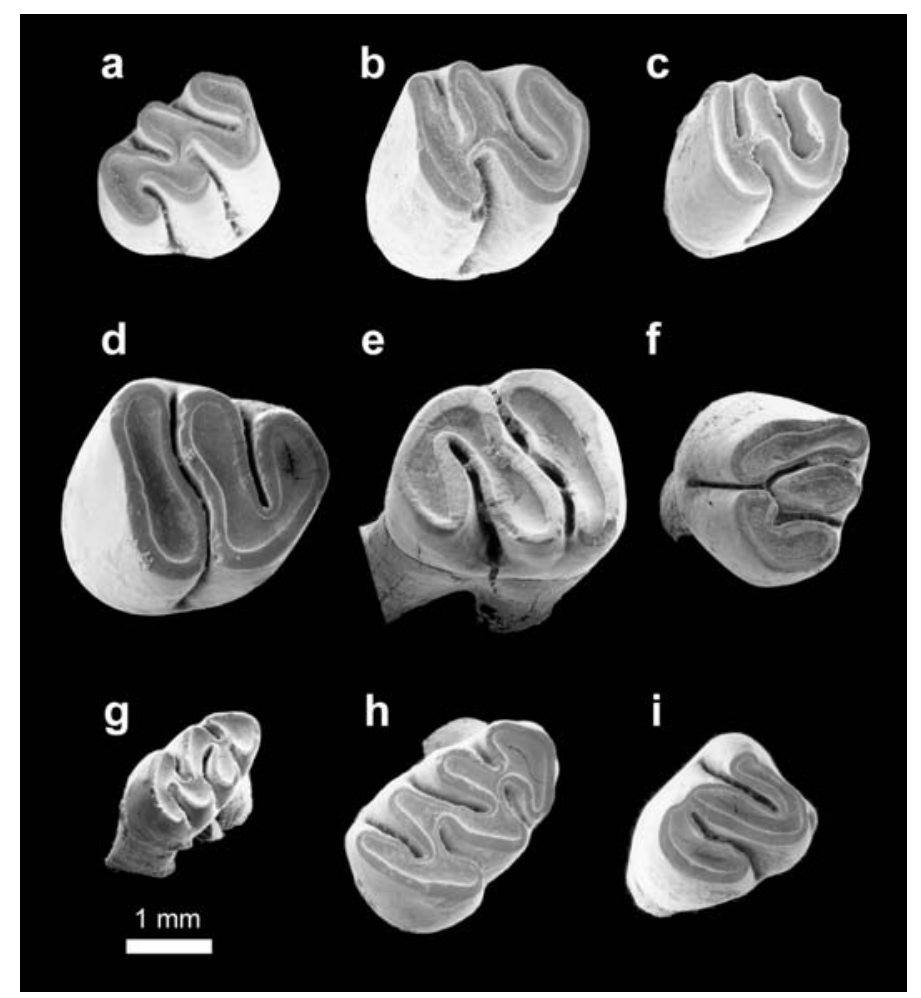

Fig. 3: Molares de Sigmodon hispidus. a: $\mathrm{M}^{1}$; b y c: corresponden a $\mathrm{M}^{2}$; d-f corresponden a ${ }^{2} \mathrm{M}$; g y h: coresponden con $\mathrm{M}^{1}$; i: $\mathrm{M}^{3}$. (Simbología como en las figuras 1 y 2). En todos los casos el desgaste de la superficie oclusal es evidente. 
ello es visible en las muestras CFM-1271 (Fig. 3a) que corresponde a un $\mathrm{M}^{1}$; CFM-1272 y 1273 (Figs. 3b y c) que corresponden a un $\mathrm{M}^{2}$, donde el posterolofo está unido al metalofo debido al desgaste de los molares y CFM-1274 y 1276 (Figs. 3d y f) que corresponden a un ${ }^{2} \mathrm{M}$, donde el posterolofo no está unido al metalofo, ello representa un grado de desgaste oclusal menor que en el caso de los $\mathrm{M}^{2}$. Los terceros molares superiores al igual que en $\operatorname{los}{ }^{2} \mathrm{M}^{2}$, ver por ejemplo CFM-1275 (Fig. $3 \mathrm{e}$ ); se caracterizan por presentar un patrón muy simple con un conspicuo posteroflexo y un hipoflexo separado del metaflexo por un muro mediano. Lo mismo sucede en los primeros molares superiores o ${ }^{1} \mathrm{M}^{1}$ donde además se observa un amplio procíngulo separado por el protoflexo y el anteroflexo (caso CFM-1271 y 1277).

Los molares inferiores se caracterizan por patrones sigmoides simples en forma de "S" muy obvio en $\operatorname{los}{ }^{2} \mathrm{M}^{2} \mathrm{y}^{3} \mathrm{M}^{3}$; por ejemplo $\mathrm{CF} ; 1278$ un $\mathrm{M}^{3}$ (Fig. 3i). En el caso de $\operatorname{los}{ }^{1} \mathrm{M}^{1}$; el patrón es muy similar al de los primeros molares superiores (Figs. 3g y h que corresponden a CFM-1279 y 1280), pero en general son más angostos en sentido vestíbulolingual.

Distribución actual: desde el norte de Venezuela y Colombia, casi todo América Central y México hasta el sureste y centro de los Estados Unidos (Kiblisky, 1969 en Cameron \& Spencer, 1981).

En Costa Rica Sigmodon hispidus se distribuye en las tierras bajas y medias de las vertientes pacífica y caribe sur, desde el nivel del mar hasta los 2700 m.s.n.m. En la vertiente caribe su distribución está asociada al bosque tropical húmedo (basado en Mc Pherson, 1985).

Distribución paleogeográfica: el género Sigmodon ha sido registrado en el Pleistoceno y Holoceno de América del Norte (Álvarez, 1964 y Martín, 1979) y de América del Sur (Fejfar et al., 1993).

La especie Sigmodon hispidus ha sido registrada para el Pleistoceno de Texas (Slaugther, 1966, 1967 en Martín, 1979); Pleistoceno Tardío de La Florida, Georgia, Louisiana y Nuevo México (Martín, 1979 y Hubert \& Prat, 1998).

En el Pleistoceno de México en Cuatro Ciénegas, estado de Coahuila (Gilmore, 1947), en la cueva de San Josecito, estado de Nuevo León (Silva Barcenas, 1969) y en la Cueva La Presita, estado de San Luis Potosí (Polaco \& Butrón, 1997).

En Costa Rica, se ha descrito para el Holoceno Medio-Tardío de la Caverna Ojos Verdes en Barra Honda, Península de Nicoya (Woodman, 1988).

Evolución de los Sigmodontinae: de acuerdo a Reig (1980), es el grupo de roedores muroideos más diversificado con 250 especies en 49-50 géneros repartidos en; América del Norte; Central y del Sur; Sigmodon hispidus es la especie común a las tres Américas. Lo interesante de este grupo es el alto endemismo sudamericano, a pesar de que no hay un grupo del Cenozoico suramericano que pueda ser postulado como el grupo ancestral de los Sigmodontinae. Es claro pues, que este grupo se originó el norte del continente a partir de roedores cricétidos del Eoceno Tardío del oeste de Norte América.

Reig (1980) sostiene que la diferenciación de los Sigmodontinae se dio en América del Sur, luego de que el puente de Panamá se estableciera, cuando un "stock" inmigrante alcanzó ese subcontinente en el Plioceno tardío; allí y de manera rápida se diversificó con una evolución marcadamente horotélica. A pesar de ello, América Central no presenta tal grado de diversificación de los Sigmodontinae, como América del Sur. Por otra parte, Reig (1984), sugiere que fue la conquista de Cordillera Andina la causante de tan rápida diversificación adaptativa.

\section{Paleoecología}

La interpretación paleoecológica de esta asociación de roedores se realiza con base en la observación de las zonas geográficas de coincidencia actual en el territorio nacional. Para dicho trabajo se contó con la colaboración de los estudiantes del programa de licenciatura en Protección y Manejo en Recursos Naturales de la Universidad Estatal a Distancia, quienes identificaron dos áreas de coincidencia geográfica (ver cuadro 1).

Como se observa en el cuadro 1, la zona de vida correspondiente al bosque pluvial premontano 
Cuadro 1

Análisis de las zonas de vida de las áreas de coincidencia actual, de las especies fósiles Tylomys watsoni, Reithrodontomys mexicanus, Sigmodon hispidus y Proechimys semispinosus

\begin{tabular}{|c|c|c|c|}
\hline $\begin{array}{l}\text { Zona de coincidencia } \\
\text { geográfica }\end{array}$ & $\begin{array}{l}\text { Altitud } \\
\text { m.s.n.m. }\end{array}$ & $\begin{array}{c}\text { Área } \\
\text { aproximada (ha) }\end{array}$ & Zona de vida \\
\hline Cercanías de San Ramón & $1100-12001$ & 475 & $\begin{array}{l}\text { Bosque muy húmedo } \\
\text { premontano } \\
\text { Bosque pluvial premontano } \\
\text { Bosque muy húmedo } \\
\text { premontano transición a pluvial }\end{array}$ \\
\hline $\begin{array}{l}\text { Cuenca media de los ríos } \\
\text { Parrita, Naranjo, Savegre, } \\
\text { Damas y Grande de Térraba }\end{array}$ & $300-1800$ & 87900 & $\begin{array}{l}\text { Las principales: } \\
\text { Bosque pluvial Premontano y } \\
\text { bosque muy húmedo } \\
\text { Premontano } \\
\text { Menor extensión: } \\
\text { Bosque muy húmedo tropical basal } \\
\text { Bosque muy húmedo transición } \\
\text { a premontano } \\
\text { Bosque tropical transición a } \\
\text { premontano }\end{array}$ \\
\hline
\end{tabular}

y bosque muy húmedo Premontano, son las zonas de vida que coinciden con el análisis paleoflorístico realizado por Pérez (2001) y Pérez \& Laurito (2003) de la localidad de La Palmera; ellos concluyeron que la flora del Pleistoceno, existente en esa localidad, es coincidente a la existente en la actualidad en un Bosque Premontano transición al Bosque Montano Bajo, equivalente al dominio altitudinal de 1200 m.s.n.m. a los 1300 m.s.n.m.

Por otra parte, todos los restos encontrados están asociados a bolos fecales y de regurgitación de aves de presa. Un análisis de los posibles predadores hecha por Corrales et al. 2002 y Chaves Chaves et al. 2002, fundamentado en datos biométricos, de comportamiento y biología de los roedores que se muestra en el cuadro 2.

De acuerdo al cuadro 2, se postulan como presuntos predadores dos especies de lechuzas, por su gran tamaño, hábitos de caza y biología son: Tyto alba y Pulsatrix perpicillata, lo que no es extraño ya que de acuerdo a Andrews (1990) son las lechuzas los mayores contribuyentes de las asociaciones fósiles de micromamíferos, en especial de roedores.

\section{CONCLUSIONES}

Se describen cuatro nuevos registros fósiles de roedores para Costa Rica: Tylomys watsoni, Reithrodontomys mexicanus, Sigmodon hispidus y Proechimys semispinosus.

Esta asociación de micromamíferos permite reforzar la paleoecología que previamente se tenía de la localidad de La Palmera de San Carlos, que a pesar de hallarse a solo 400 m.s.n.m., coincide con la zona de vida actual Bosque Premontano transición al Bosque Montano Bajo, equivalente al dominio altitudinal de 1200 m.s.n.m. a los 1300 m.s.n.m. Ello permite concluir un ambiente mucho más frío, lo que a su vez sugiere una edad probable Pleistoceno Superior para el yacimiento de La Palmera.

Todos los roedores encontrados, viven en la actualidad en el territorio nacional, pero solo dos especies Proechimys semispinosus y Tylomys watsoni han sido reportados para la localidad de La Palmera (Mc Pherson, 1985).

También se postulan dos especies probables de depredadores de los roedores, las lechuzas Tyto alba y Pulsatrix perpicillata, por su 


\section{Cuadro 2}

Datos biométricos, de hábitats y etológicos de Tylomys watsoni, Reithrodontomys mexicanus, Sigmodon hispidus y Proechimys semispinosus

\begin{tabular}{|c|c|c|c|c|c|}
\hline & & Longitud (mm) & Peso (gr) & Habitat & Conducta \\
\hline \multirow{4}{*}{ 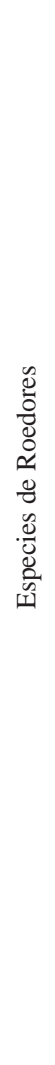 } & $\begin{array}{l}\text { Tylomys } \\
\text { watsoni }\end{array}$ & $\begin{array}{l}\text { Cuerpo:155-290 } \\
\text { Cola: } 150-320\end{array}$ & $196-252$ & $\begin{array}{l}\text { Bosques húmedos } \\
\text { densos primarios y } \\
\text { secundarios }\end{array}$ & $\begin{array}{l}\text { Nocturno } \\
\text { Semiarborícola, } \\
\text { visita el suelo en } \\
\text { periodos breves, } \\
\text { rondan cuevas, } \\
\text { áreas rocosas y } \\
\text { troncos caídos }\end{array}$ \\
\hline & $\begin{array}{l}\text { Sigmodon } \\
\text { hispidus }\end{array}$ & $\begin{array}{l}\text { Cuerpo: } 112-160 \\
\text { Cola: } 88-130\end{array}$ & $38-87$ & $\begin{array}{l}\text { Bosques secos y } \\
\text { húmedos, sabanas } \\
\text { arboladas, charrales, } \\
\text { plantaciones de caña } \\
\text { y palma africana } \\
\text { asociada a pastizales }\end{array}$ & Diurno \\
\hline & $\begin{array}{l}\text { Reithrodontomys } \\
\text { mexicanus }\end{array}$ & $\begin{array}{l}\text { Cuerpo: } 69-100 \\
\text { Cola: } 92-134\end{array}$ & $14-18$ & $\begin{array}{l}\text { Bosques húmedo } \\
\text { riparios, secundarios } \\
\text { con árboles altos }\end{array}$ & $\begin{array}{l}\text { Nocturno } \\
\text { Semiarborea, Vive } \\
\text { en cavidades de } \\
\text { troncos, arbustos } \\
\text { de follaje denso }\end{array}$ \\
\hline & $\begin{array}{l}\text { Proechimys } \\
\text { semispinosus }\end{array}$ & $\begin{array}{l}\text { Cuerpo: }>350 \\
\text { Cola: }>150\end{array}$ & $320-536$ & $\begin{array}{l}\text { Bosques } \\
\text { siempre verdes, } \\
\text { Bosques húmedo } \\
\text { Bosques secundarios, } \\
\text { bosques deciduos con } \\
\text { corredores que } \\
\text { conectan a zonas riparias }\end{array}$ & $\begin{array}{l}\text { Nocturno y } \\
\text { principalmente } \\
\text { terrestre, caminan } \\
\text { en troncos huecos } \\
\text { pero no suben a } \\
\text { los árboles,viven } \\
\text { en cuevas que } \\
\text { excavan en el } \\
\text { suelo o en } \\
\text { depresiones bajo } \\
\text { las raíces }\end{array}$ \\
\hline \multirow{2}{*}{ 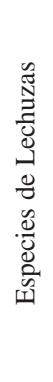 } & Tyto alba & 40 & 425 & $\begin{array}{l}\text { Áreas abiertas con } \\
\text { árboles esparcidos, } \\
\text { zonas agrícolas, } \\
\text { pantanos abiertos y } \\
\text { bancos de lodo }\end{array}$ & $\begin{array}{l}\text { Nocturno. A veces } \\
\text { caza al amanecer y } \\
\text { al anochecer }\end{array}$ \\
\hline & $\begin{array}{l}\text { Pulsatrix } \\
\text { perpicillata }\end{array}$ & 48 & 750 & $\begin{array}{l}\text { Bosques densos. } \\
\text { Caza también a lo } \\
\text { largo de los bordes, } \\
\text { en los claros o en } \\
\text { sabanas con } \\
\text { árboles grandes }\end{array}$ & $\begin{array}{l}\text { Nocturno, a veces } \\
\text { caza de día }\end{array}$ \\
\hline
\end{tabular}

Basado en Carrillo et al. 1999; Emmons, 1999, Méndez, 1993; Reid, 1997 y Stiles \& Skutch,1998 en Chaves et al. 2002 y Corrales Leitón et al. 2002. 
gran tamaño y su capacidad de levantar vuelo con especies tan grandes como Proechimys semispinosus; además de tener hábitos diurnos y nocturnos, lo que les permite cazar, aparte de especies nocturnas como Tylomys watsoni, Reithrodontomys mexicanus y Proechimys semispinosus, especies diurnas como Sigmodon hispidus.

\section{AGRADECIMIENTOS}

Al Lic. Eduardo A. Pérez Gamboa, por ayudar en la recolecta de los fósiles y permitirme el acceso a la colección paleobotánica, además del aporte de sus valiosos comentarios e ideas.

Al señor Bernal Rodríguez, mastozoólogo y curador de la colección de roedores del Museo Nacional de Costa Rica, cuyo apoyo solidario permitió llevar a buen fin la confirmación de las especies de roedores.

A los alumnos del curso especializado $\mathrm{Pa}$ leobiogeografía código 09555 de la Universidad Estatal a Distancia, año 2002, que han hecho posible el desarrollo de esta investigación.

A la Unidad de Microscopía Electrónica de la Universidad de Costa Rica, quienes realizaron las imágenes que ilustran este trabajo.

Al Dr. Arroyo Cabrales del Instituto Nacional de Antropología e Historia de México y a los Drs. Lorenzo Rook; Marco Ferretti y Giuseppe Tito de la Universtita degli Studi di Firenze, quienes gentilmente cooperaron con parte de la bibliografía.

\section{REFERENCIAS}

ÁLVAREZ, T., 1964: Nota sobre restos óseos de mamíferos del Reciente, encontrados cerca de Tepeapulco, Hidalgo, México. - 16 págs. Publ. Depto. de Prehist., Inst. Nac. de Antropol. e Hist. 15.

ANDREWS, P., 1990: Owls, caves and fossils. - 231 págs. Univ. of Chicago Press.

CAMERON, G.N \& SPENCER, S.R., 1981: Sigmodon hispidus. - Mammalian Species 158: 1-9.
CHAVES, G.; LOBO, S.; MEDINA, W. MONTERO, V. \& SEVILLA, L., 2002: Identificación y descripción de roedores a partir de fósiles colectados en La Palmera de San Carlos, Alajuela, Costa Rica. - 56 págs. Escuela de Ciencias Exactas y Naturales, UNED [Inf. inédito].

CORRALES, A.L.; GAITÁN, M.; MESÉN, G.; PICADO, N. \& SANDÍ, E., 2002: Identificación de especies de roedores, la biología, ecología e interpretación ambiental de Tylomys watsoni, Reithrodontomys mexicanus, Sigmodon hispidus y Proechimys semispinosus. - 44 págs. Escuela de Ciencias Exactas y Naturales, UNED [Inf. inédito].

FEJFAR, O.; BLASSETTI, A.; CALDERONI, G.; COLTORTI, M.; FICCARELLI, G.; MASINI, F; ROOK \& TORRE, D., 1993: New finds of cricetids (Mammalia, Rodentia) from the late Pleistocene-Holocene of northern Ecuador. - Docum. Lab. Géol. Lyon, 125: 151-167.

GILMORE, R.M., 1947: Report on a collection of mammals bones from archeological cave sites in Coahuila, Mexico. - J. Mammalogy 28(2): 147-165.

HUBERT; R.C. \& PRAT, A.E., 1998: New Pleistocene (Rancholabrean) vertebrate faunas from coastal Georgia. - J. Vertebrate Paleontol. 18(2): 412-429.

Mc PHERSON, A.B., 1985: A biogeographical analysis of factors influencing the distribution of Costa Rica Rodents. - Brenesia 23: 97-273.

MARTIN, R.A., 1979: Fossil history of the rodent genus Sigmodon. - Evolutionary Monographs 2: 1-36.

PÉREZ, E.A., 2001: Comparación de la flora fósil del Pleistoceno con la flora actual en la localidad de La Palmera de San Carlos, Provincia de Alajuela. - 126 págs. Escuela de Ciencias Exactas y Naturales, Universidad Estatal a Distancia [Tesis Lic.].

PÉREZ, E.A., 2004: Presencia de Juglans olanchana Standley \& Williams (Juglandaceae) en territorio costarricense durante el Pleistoceno. - Rev. Geol. Amer. Central, 28: 77-81.

PÉREZ, E.A. \& LAURITO, C.A., 2004: Quercus corrugata Hooker (Fagacea) como indicador paleoclimático del Pleistoceno de Costa Rica. - Rev. Geol. Amer. Central, 28:83-90.

POLACO, O.J. \& BUTRÓN, L., 1997: Mamíferos pleistocénicos de la cueva La Presita, San Luis Potosí, México. En: ARROYO, J. \& POLACO, O.J. (eds.): Homenaje 
al Profesor Ticul Álvarez. Instituto Nacional de Antropología e Historia, México: 279-296.

REID, F.A., 1997: A field guide to the mammals of Central America and southern Mexico. - 337 págs. Oxford University Press.

REIG, O.A., 1980: A new fossil genus of South America cricetid rodents allied to Wiedomys, with an assessment of the Sigmodontinae. - J. Zool. Lond. 192: $257-281$
REIG, O.A., 1984: Distribuçâo geográfica e história evolutiva dos roedores muroideos sulamericanos (Cricetidae: Sigmodontinae). - Rev. Brasil. Genet. 7(2): 333-365.

SILVA BARCENAS, A., 1969: Localidades de vertebrados fósiles en la república mexicana. - Paleont. Mexicana 28: 34 págs.

WOODMAN, N., 1988: Subfosil remains of Peromyscus stirtoni (Mammalia: Rodentia) from Costa Rica. - Rev. Biol. Trop. 36(2A): 247-253. 